\title{
A national survey of transition from pediatric to adult healthcare providers for adolescents and young adults with type 1 diabetes: perspectives of pediatric endocrinologists in Korea
}

Jae Hyun Kim ${ }^{1,2}$,

Jae-Ho Yoo ${ }^{3}$

'Department of Pediatrics, Seoul National University Bundang Hospital, Seongnam, Korea

${ }^{2}$ Department of Pediatrics, Seoul National University College of Medicine, Seoul, Korea

${ }^{3}$ Department of Pediatrics, Dong-A University College of Medicine, Busan, Korea

Received: 25 August, 2020

Accepted: 6 September, 2020

Address for correspondence: Jae-Ho Yoo

Department of Pediatrics, Dong-A University College of Medicine, 32 Daesingongwon-ro, Seo-gu, Busan 49201, Korea

Email: pedendo@dau.ac.kr https://orcid.org/0000-0003-3065944X
Purpose: Transition from pediatric to adult healthcare for adolescents and young adults with type 1 diabetes (T1D) increases the risk for poor outcomes. This study aimed to describe the circumstances and clinical practice patterns associated with this transition of care based on a nationwide survey of pediatric endocrinologists in Korea.

Methods: An electronic survey regarding the healthcare transition of T1D patients was administered to 143 pediatric endocrinologists registered in the Korean Society of Pediatric Endocrinology.

Results: The response rate was $50.2 \%(n=72)$. Among responders, $58.3 \%(n=42)$ were females, and $70.8 \%(n=51)$ worked in academic medical centers. The main reasons for transfer to adult care were request from a patient or family $(69.6 \%)$ and age $\geq 18$ years $(42.0 \%)$. Impediments to transition were a longlasting therapeutic relationship (72.9\%) and lack of adult specialists in T1D care (62.9\%). Communication between pediatric and adult endocrinologists was via nonstructured patient summary (68.6\%) and telephone or email (27.1\%). Responders reported that successful transition requires development of transition protocols (79.2\%) and a multidisciplinary team approach for transition care (52.8\%). Conclusion: Transition care of T1D patients is a challenge to pediatric endocrinologists in Korea. Development of transition care protocols for healthcare providers and improvement of diabetes self-management skills for patients are needed.

Keywords: Type 1 diabetes mellitus, Transition care, Adolescents, Young adults, Korea

\section{Highlights}

Transition care of T1D patients was a considerable challenge to pediatric endocrinologists. This national survey indicated many impediments for transition care in pediatric providers in Korea. Development of protocols for transition care is required to successful transition.

\section{Introduction}

Type 1 diabetes (T1D) is one of the most common chronic diseases in childhood. ${ }^{1,2)}$ Longterm management of the disease from childhood to adulthood is essential. ${ }^{3,4)}$ Along the course of disease management, there is a time to transfer from pediatric to adult healthcare system. The transition period corresponds to emerging adulthood, generally from the late teens to early twenties. ${ }^{5}$ T1D patients in the transition period are associated with unfavorable health 
outcomes, including poor glycemic control, loss of followup, psychological problems, and increased acute and chronic complications. $^{6-10)}$ Therefore, proper management in the transition period is critical for improving clinical outcomes in T1D patients.

For management of T1D patients in the transition period, the American Diabetes Association and other academic societies published a position statement in 2011, providing recommendations for transition care for pediatric providers and emerging adults with T1D. ${ }^{11)}$ In several guidelines and reviews, successful transition is an indispensable part of T1D care. ${ }^{12-}$

15) Although the Endocrine Society and other professional societies have developed several transition toolkits for T1D, it is uncertain whether these guidelines are well applied in clinical practice. ${ }^{16)}$ Moreover, there are no widely accepted guidelines or protocols for transition care in T1D patients.

To date, many studies have focused on issues related to patients/family of T1D patients, ${ }^{17-20)}$ adult providers, ${ }^{21,22)}$ and pediatric providers, ${ }^{23,24)}$ especially in Western countries. However, studies on Korean patients are sparse ${ }^{25)}$ because the incidence of T1D in children and adolescents in Korea has been low, although increasing incidence has been observed. ${ }^{26,27)}$ Moreover, studies on transition care of T1D patients from the point of view of pediatric providers have not been carried out.

The aim of this study was to describe clinical practice characteristics and circumstances, investigate the reasons and impediments to T1D transition care, and identify clinical practice patterns of pediatric endocrinologists associated with transition care of patients with T1D, from the perspective of pediatric providers using a nationwide survey.

\section{Materials and methods}

\section{Survey design}

A structured questionnaire was designed based on practice circumstances of Korea and previous studies. ${ }^{22,24,28)}$ The questionnaire was comprised of three parts with 16 questions: (1) characteristics of responders including age, sex, and duration of practice as a pediatric endocrinologist; (2) practice circumstances including practice setting (primary, secondary, or tertiary center), number of patients with T1D, percentage of T1D patients aged 18 years or more, and composition of pediatric diabetes care team; and (3) process of transition such as reasons for transition, glycemic control at the time of transition, communication and feedback with adult endocrinologists, impediments to transition to adult clinic, and suggestions for improvement of transition care. The responses were in the form of "single most important answer" and "all relevant answers."

\section{Data collection}

Candidates for the survey were 143 pediatric endocrinologists in Korea who were registered in the Korean Society of Pediatric Endocrinology (KSPE) at the time of survey. Electronic survey was conducted using SurveyMonkey (Momentive Global Inc., Anaheim, CA, USA). An email embedded with the survey link was sent to all members of the KSPE on April 17, 2017. Data collected until April 30, 2017 were used for the analysis. Responses were anonymous, and there was no incentive to participate in the survey.

\section{Statistical analysis}

Data were analyzed using Stata 16.0 (StataCorp LP, College Station, TX, USA). Data were expressed as mean \pm standard deviation for continuous variables and number and percentage for categorical variables. Student $t$-tests and Fisher exact tests were used for comparison of continuous and categorical variables, respectively. A $P$-value of $<0.05$ was considered statistically significant.

\section{Results}

\section{Characteristics and practice circumstances of respon- ders}

The survey response rate was $50.2 \%(n=72)$. Characteristics of responders are described in Table 1 . Among responders, females were predominant $(n=42,58.3 \%)$. Practice duration as a pediatric endocrinologist was $13.0 \pm 8.9$ years. Proportion of responders aged between 31 and 50 years was $75 \%(n=54)$. Fiftyone responders $(70.8 \%)$ worked in tertiary centers. Number of patients with T1D varied, although $65.4 \%$ of responders followed $\leq 50$ patients. Most responders $(84.7 \%)$ had $\leq 25 \%$ of T1D patients aged $\geq 18$ years.

Male respondents had a longer practice duration (15.8 years vs. 11.3 years, $P=0.038$ ) and more patients with T1D $(P=0.041)$. There were no differences in sex and practice duration according to practice setting $(P=0.867$ and $P=0.445$, respectively). There was a tendency of increasing number of diabetes care team members (2.5 in primary, 3.1 in secondary, and 4.0 in tertiary center; $P=0.007)$. The number of T1D patients was relatively high in tertiary centers $(P=0.002)$.

\section{Reasons and glycemic control in transition}

All relevant reasons for transfer to adult clinic were patient/ family request (69.6\%), age $\geq 18$ years $(42.0 \%)$, development of comorbidity or diabetes complications (34.8\%), move to another city/village (30.4\%), and admission to university (29.0\%) (Fig. 1). The reasons for transfer were ranked as follows: patient/ family request (40.0\%), development of comorbidity or diabetes complications ( $18.6 \%)$, age $\geq 18$ years $(17.1 \%)$, move to another city/village (7.1\%), and better glycemic control (5.7\%).

With regard to glycemic control during transition, responses 
Table 1. Characteristics of pediatric endocrinologists and their practice $(n=72)$

\begin{tabular}{|c|c|}
\hline Variable & Value \\
\hline \multicolumn{2}{|l|}{ Age (yr) } \\
\hline $31-40$ & $27(37.5)$ \\
\hline $41-50$ & $27(37.5)$ \\
\hline $51-60$ & $12(16.7)$ \\
\hline$\geq 61$ & $6(83)$ \\
\hline \multicolumn{2}{|l|}{ Sex } \\
\hline Male & $30(41.7)$ \\
\hline Female & $42(58.3)$ \\
\hline Practice duration $(y r)(n=71)$ & $13.0 \pm 8.9$ \\
\hline \multicolumn{2}{|l|}{ Practice setting } \\
\hline Primary & $2(2.8)$ \\
\hline Secondary & $19(26.4)$ \\
\hline Tertiary & $51(70.8)$ \\
\hline \multicolumn{2}{|c|}{ No. of patients of type 1 diabetes } \\
\hline$>200$ & $6(8.3)$ \\
\hline $101-200$ & $6(8.3)$ \\
\hline $51-100$ & $13(18.1)$ \\
\hline $26-50$ & $22(30.6)$ \\
\hline$\leq 25$ & $25(34.7)$ \\
\hline \multicolumn{2}{|c|}{ Percentage of type 1 diabetes patients aged $\geq 18$ years } \\
\hline$>50 \%$ & $2(2.8)$ \\
\hline $26 \%-50 \%$ & $9(12.5)$ \\
\hline $11 \%-25 \%$ & $23(31.9)$ \\
\hline $6 \%-10 \%$ & $13(18.1)$ \\
\hline $1 \%-5 \%$ & $16(22.2)$ \\
\hline $0 \%$ & $9(12.5)$ \\
\hline \multicolumn{2}{|c|}{ Health care providers in practice } \\
\hline Pediatric endocrinologist & $72(100)$ \\
\hline Dietitian & $67(93.1)$ \\
\hline Nurse & $57(79.2)$ \\
\hline Mental health specialist & $16(22.2)$ \\
\hline Social worker & $42(58.3)$ \\
\hline Physical educator & $12(16.7)$ \\
\hline Coordinator & $2(2.8)$ \\
\hline
\end{tabular}

Values are presented as number (\%) or mean \pm standard deviation.

varied. T1D patients in transition were preferentially transferred to an adult clinic with very good glycemic control (glycosylated hemoglobin $[\mathrm{HbAlc}]<7.0 \%$ [53 mmol/mol], 22.9\%), good glycemic control (HbAlc 7.0\%-8.5\% [53-69 mmol/mol], $30.0 \%$ ), poor glycemic control (HbAlc 8.6\%-10.0\% [70-86 $\mathrm{mmol} / \mathrm{mol}], 12.9 \%$ ), and very poor glycemic control ( $\mathrm{HbAlc}$ $>10.0 \%[86 \mathrm{mmol} / \mathrm{mol}], 34.3 \%)$. There was no difference in glycemic control at transfer by sex and practice setting (all $P>0.05)$.

\section{Communication with adult endocrinologists}

Pediatric endocrinologists in Korea communicated with adult endocrinologists via unstructured patient summary (68.6\%), telephone or email (27.1\%), overlap of pediatric and adult practice $(12.9 \%)$, structured document $(7.1 \%)$, or without

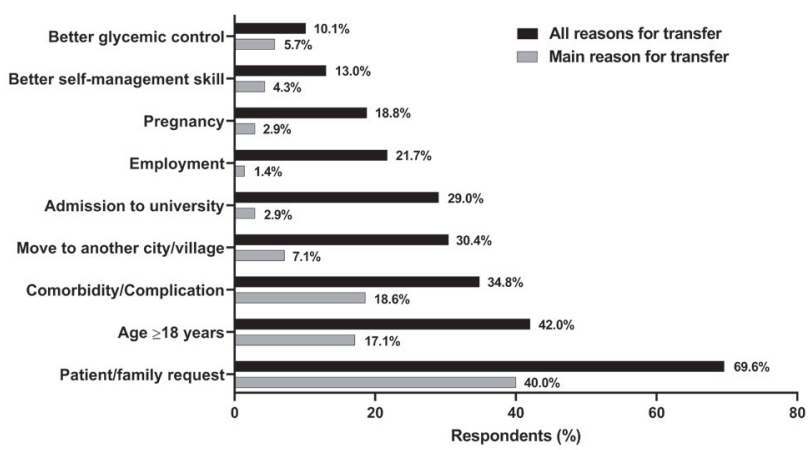

Fig. 1. Reasons for transfer to adult clinic.

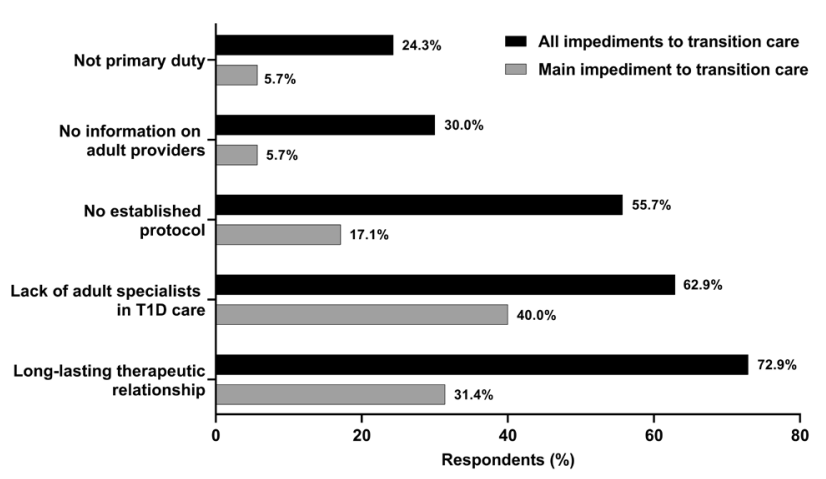

Fig. 2. Impediments to transition care. T1D, type 1 diabetes.

any document or contact (12.9\%) at the time of transition. After transfer, feedback from adult endocrinologists was by way of telephone (10.1\%), letter or email (10.1\%), or regular feedback (1.4\%). However, $79.7 \%$ of respondents did not receive any feedback.

\section{Impediments to transition care}

For pediatric endocrinologists, impediments to transition care were long-lasting therapeutic relationship with patient/ family (72.9\%), lack of adult specialists in T1D care (62.9\%), no established protocol for transition care (55.7\%), no information on adult providers (30.0\%), and not having enough time because of low priority (24.3\%) (Fig. 2). Forty percent of respondents considered lack of adult specialist in T1D care the most important impediment to transition care, followed by longlasting therapeutic relationship with patient/family (31.4\%) and no established protocol for transition care (17.1\%). There was no difference in sex or age by impediment (all $P>0.05$ )

\section{Suggestions for effective transition care}

For effective transition care, respondents suggested the need for guidelines for transition care $(72.9 \%)$, multidisciplinary team care with patient/family as well as pediatric and adult providers (52.8\%), education of adult and pediatric providers 


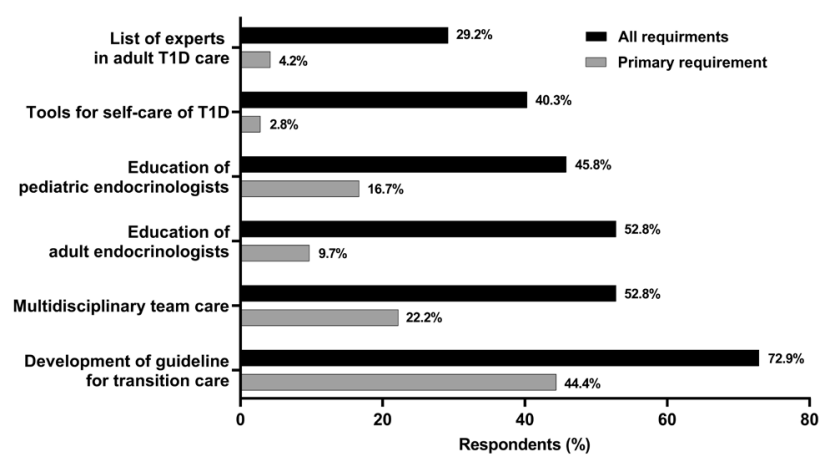

Fig. 3. Requirements for effective transition care. T1D, type 1 diabetes.

(52.8\% and $45.8 \%$, respectively), development of tools for selfcare evaluation of T1D (40.3\%), and provision of a list of experts for adult T1D care (29.2\%) (Fig. 3). Among them, development of guidelines for transition care (44.4\%) was considered most important.

\section{Discussion}

To the best of our knowledge, the present study is the first on transition care from the perspective of pediatric providers in Korea and Asia, where the incidence of T1D is low. In this nationwide survey with a high response rate, pediatric endocrinologists in Korea indicated that patient/family request was the main reason for transfer to adult clinic, and lack of adult specialists in T1D care and long-lasting therapeutic relationship with patient/family were the main impediments to transition care. Pediatric providers in Korea mostly communicated with adult using unstructured documents, although almost $80 \%$ of the respondents did not receive any feedback after transfer. For effective transition care, pediatric endocrinologists in Korea considered development of guidelines for transition care as the most important factor.

The transition period in T1D patients is associated with unfavorable health outcome. ${ }^{6-10)}$ Transition indicates "planned and purposeful shift from a child- to adult-centered healthcare system." ${ }^{29)}$ Therefore, for successful transition and to reduce transition-related health problems, preparation for transition via participation of patient/family and pediatric and adult care providers is of paramount importance. Several guidelines have recommended that pediatric providers should start preparing for transition at least one year before transfer, although the appropriate age at transfer is unclear. ${ }^{11,14)}$ In the present study, two main reasons to embark on transition for pediatric endocrinologists in Korea were patient/family request and age $\geq 18$ years, which were similar to another study. ${ }^{24)}$ Moreover, onethird of the respondents identified development of comorbidity or diabetes complications as reasons for transfer; pediatric providers can be unfamiliar with management of these complications. These responses imply that transition in Korea is a relatively passive but abruptly occurring process driven by outside circumstances rather than by diabetes team and patient/ family engagement.

Diabetes self-management skills are key requirements for successful transition. A recent study showed that good executive functioning skills and balanced responsibility for diabetes management tasks between youth and parents were associated with better glycemic control around the transition period. ${ }^{30)}$ Therefore, during preparation for transition, education of T1D patients should focus on the gradual shift of care responsibility from the family to patients. However, in the present study, only $4.3 \%$ of respondents answered that better self-management skills were a primary reason for transfer. There is a wide gap between guidelines and real-world practice, and measures to fill this gap are necessary.

Impediments to transition care in this survey were termination of long-lasting therapeutic relationship with patient/family, lack of adult specialists in T1D care, and absence of established protocols. With regard to ending long-term relationship, no sex difference was observed in the present study, whereas female predominance was noted in another study. ${ }^{24)} \mathrm{T} 1 \mathrm{D}$ patients in Korea were managed by pediatric endocrinologists in hospitals rather than primary care physicians, because the incidence of T1D in childhood is very low and typically is diagnosed in hospitals. Therefore, pediatric patients with T1D tended to be transferred to adult endocrinologists in the same hospital, unless they were moving to another place or proceeding to university. However, due to a high prevalence of type 2 diabetes among adults in Korea, adult providers are mainly engaged in management of type 2 diabetes. As a result, it seems to be difficult to find adult specialists for T1D care in Korea. This might be the same in countries with a low incidence of T1D in childhood. Thus, this study highlights the challenges of low T1D incidence on transition care. Providing a list of adult experts for T1D care at the professional medical society level might be a countermeasure to tackle the lack of information.

Most pediatric endocrinologists in Korea communicated with adult counterparts via unstructured documents. However, adult care providers in the United States reported that patient records and summaries were an integral part of healthcare transition. ${ }^{22)}$ Therefore, development of structured documents with essential information for transition is necessary. Contrary to contact with adult endocrinologists around the time of transition in almost $90 \%$ of pediatric providers, approximately $80 \%$ of them did not receive any feedback from their adult counterparts after transfer. At transition, clinic attendance rate is associated with glycemic control. ${ }^{19,31)}$ To prevent missed attendance after transfer, mutual communication between pediatric and adult providers over the transition period might be required.

Korean pediatric endocrinologists considered development of guidelines as most important for an effective transition. In a Canadian study, a structured program for transition was helpful in improving clinical attendance and decreasing diabetes-associated distress. ${ }^{32)}$ Transition process varied by clinic. ${ }^{23)}$ Moreover, transition is influenced by psychosocial development of patient and family background. Therefore, 
culturally-sensitive and well-organized guidelines with detailed instructions are necessary. In addition, a multidisciplinary team approach including pediatric and adult providers is critical for preparation of a transition, which promotes diabetes self-care skills of patients and duties as emerging adults.

Many pediatric endocrinologists responded that education programs on transition care for both pediatric and adult care providers were necessary for effective transition. Training for transition care influenced several aspects such as smooth transition process, well-organized documentation, and less frequent return to the pediatric clinic. ${ }^{24)}$ In the present study, reception of transition education was not determined. However, such training is not common in Korea, and efforts to setup transition training programs are needed.

The present study had limitations. Almost half of the KSPE members did not respond to the survey request. However, the result of the study might reflect the situation in Korea since most doctors working in hospitals answered the survey. The survey also excluded adult endocrinologists, patients, and family members, who might have had different points of view on transition. However, this is the first study to evaluate the perspectives of pediatric endocrinologists on transition care in Korea and Asia, where the incidence of T1D is low.

In conclusion, our findings revealed that transition care of T1D patients was a considerable challenge to pediatric endocrinologists in Korea. This national survey indicated that development of a culturally sensitive protocol for transition care, multidisciplinary team approach, and education for pediatric and adult care providers are required for effective transition of T1D patients. Moreover, preparation for transition should be directed at improving self-management skills of emerging adults. In addition, unmet needs of adult care providers and patient/family should be investigated.

\section{Ethical statement}

This study was approved by the Institutional Review Board (IRB) of Seoul National University Bundang Hospital (IRB No. X-1711-435-904).

\section{Conflict of interest}

No potential conflict of interest relevant to this article was reported.

\section{Acknowledgments}

The authors appreciate the members of the Korea Society of Pediatric Endocrinology who participated in the survey for the present study.

\section{References}

1. DiMeglio LA, Evans-Molina C, Oram RA. Type 1 diabetes.
Lancet 2018;391:2449-62.

2. Diaz-Valencia PA, Bougneres P, Valleron AJ. Global epidemiology of type 1 diabetes in young adults and adults: a systematic review. BMC Public Health 2015;15:255.

3. Chiang JL, Maahs DM, Garvey KC, Hood KK, Laffel LM, Weinzimer SA, et al. Type 1 diabetes in children and adolescents: a position statement by the American Diabetes Association. Diabetes Care 2018;41:2026-44.

4. Chiang JL, Kirkman MS, Laffel LM, Peters AL, Type 1 Diabetes Sourcebook A. Type 1 diabetes through the life span: a position statement of the American Diabetes Association. Diabetes Care 2014;37:2034-54.

5. Arnett JJ. Emerging adulthood. A theory of development from the late teens through the twenties. Am Psychol 2000;55:469-80.

6. Kipps S, Bahu T, Ong K, Ackland FM, Brown RS, Fox CT, et al. Current methods of transfer of young people with type 1 diabetes to adult services. Diabet Med 2002;19:649-54.

7. Bryden KS, Dunger DB, Mayou RA, Peveler RC, Neil HA. Poor prognosis of young adults with type 1 diabetes: a longitudinal study. Diabetes Care 2003;26:1052-7.

8. Busse FP, Hiermann P, Galler A, Stumvoll M, Wiessner T, Kiess W, et al. Evaluation of patients' opinion and metabolic control after transfer of young adults with type 1 diabetes from a pediatric diabetes clinic to adult care. Horm Res 2007;67:132-8.

9. Weissberg-Benchell J, Wolpert H, Anderson BJ. Transitioning from pediatric to adult care: a new approach to the postadolescent young person with type 1 diabetes. Diabetes Care 2007;30:2441-6.

10. Laing SP, Jones ME, Swerdlow AJ, Burden AC, Gatling W. Psychosocial and socioeconomic risk factors for premature death in young people with type 1 diabetes. Diabetes Care 2005;28:1618-23.

11. Peters A, Laffel L, American Diabetes Association Transitions Working Group. Diabetes care for emerging adults: recommendations for transition from pediatric to adult diabetes care systems: a position statement of the American Diabetes Association, with representation by the American College of Osteopathic Family Physicians, the American Academy of Pediatrics, the American Association of Clinical Endocrinologists, the American Osteopathic Association, the Centers for Disease Control and Prevention, Children with Diabetes, The Endocrine Society, the International Society for Pediatric and Adolescent Diabetes, Juvenile Diabetes Research Foundation International, the National Diabetes Education Program, and the Pediatric Endocrine Society (formerly Lawson Wilkins Pediatric Endocrine Society). Diabetes Care 2011;34:2477-85.

12. American Academy of Pediatrics, American Academy of Family Physicians, American College of Physicians, Transitions Clinical Report Authoring Group, Cooley WC, Sagerman PJ. Supporting the health care transition from adolescence to adulthood in the medical home. Pediatrics 
2011;128:182-200.

13. Cameron FJ, Garvey K, Hood KK, Acerini CL, Codner E. ISPAD Clinical Practice Consensus Guidelines 2018: diabetes in adolescence. Pediatr Diabetes 2018;19 Suppl 27:250-61.

14. American Diabetes Association. 13. Children and adolescents: standards of medical care in diabetes-2020. Diabetes Care 2020;43:S163-82.

15. Lee YA. Diabetes care for emerging adults: transition from pediatric to adult diabetes care systems. Ann Pediatr Endocrinol Metab 2013;18:106-10.

16. The Endocrine Society. Transition of care, type 1 diabetes [Internet]. Washington, DC; Endocrine Society; 2021 [cited 2020 May 1]. Available from: https://www.endocrine.org/ improving-practice/patient-resources/transitions/t1d.

17. Garvey KC, Beste MG, Luff D, Atakov-Castillo A, Wolpert HA, Ritholz MD. Experiences of health care transition voiced by young adults with type 1 diabetes: a qualitative study. Adolesc Health Med Ther 2014;5:191-8.

18. Kellett J, Sampson M, Swords F, Murphy HR, Clark A, Howe A, et al. Young people's experiences of managing type 1 diabetes at university: a national study of UK university students. Diabet Med 2018;35:1063-71.

19. Fisher E, Lazar L, Shalitin S, Yackobovitch-Gavan M, de Vries L, Oron T, et al. Association between glycemic control and clinic attendance in emerging adults with type 1 diabetes: a tertiary center experience. J Diabetes Res 2018;2018:9572817.

20. Garvey KC, Foster NC, Agarwal S, DiMeglio LA, Anderson BJ, Corathers SD, et al. Health care transition preparation and experiences in a U.S. National Sample of young adults with type 1 diabetes. Diabetes Care 2017;40:317-24.

21. Lyons SK, Helgeson VS, Witchel SF, Becker DJ, Korytkowski MT. Physicians' self-perceptions of care for emerging adults with type 1 diabetes. Endocr Pract 2015;21:903-9.

22. Garvey KC, Telo GH, Needleman JS, Forbes P, Finkelstein JA, Laffel LM. Health care transition in young adults with type 1 diabetes: perspectives of adult endocrinologists in the U.S. Diabetes Care 2016;39:190-7.

23. de Beaufort C, Jarosz-Chobot P, Frank M, de Bart J, Deja G. Transition from pediatric to adult diabetes care: smooth or slippery? Pediatr Diabetes 2010;11:24-7.
24. Agarwal S, Garvey KC, Raymond JK, Schutta MH. Perspectives on care for young adults with type 1 diabetes transitioning from pediatric to adult health systems: a national survey of pediatric endocrinologists. Pediatr Diabetes 2017;18:524-31.

25. Kim G, Choi EK, Kim HS, Kim H, Kim HS. Healthcare transition readiness, family support, and self-management competency in Korean emerging adults with type 1 diabetes mellitus. J Pediatr Nurs 2019;48:e1-7.

26. Lee JH, Kim YM, Kwak MJ, Kim SY, Kim HJ, Cheon CK, et al. Incidence trends and associated factors of diabetes mellitus in Korean children and adolescents: a retrospective cohort study in Busan and Gyeongnam. Ann Pediatr Endocrinol Metab 2015;20:206-12.

27. Kim JH, Lee CG, Lee YA, Yang SW, Shin CH. Increasing incidence of type 1 diabetes among Korean children and adolescents: analysis of data from a nationwide registry in Korea. Pediatr Diabetes 2016;17:519-24.

28. Garvey KC, Wolpert HA, Laffel LM, Rhodes ET, Wolfsdorf JI, Finkelstein JA. Health care transition in young adults with type 1 diabetes: barriers to timely establishment of adult diabetes care. Endocr Pract 2013;19:946-52.

29. Blum RW, Garell D, Hodgman CH, Jorissen TW, Okinow NA, Orr DP, et al. Transition from child-centered to adult health-care systems for adolescents with chronic conditions. A position paper of the Society for Adolescent Medicine. J Adolesc Health 1993;14:570-6.

30. Vloemans AF, Eilander MMA, Rotteveel J, Bakker-van Waarde WM, Houdijk E, Nuboer R, et al. Youth with type 1 diabetes taking responsibility for self-management: the importance of executive functioning in achieving glycemic control: results from the longitudinal DINO study. Diabetes Care 2019;42:225-31

31. White M, O'Connell MA, Cameron FJ. Clinic attendance and disengagement of young adults with type 1 diabetes after transition of care from paediatric to adult services (TrACeD): a randomised, open-label, controlled trial. Lancet Child Adolesc Health 2017;1:274-83.

32. Spaic T, Robinson T, Goldbloom E, Gallego P, Hramiak I, Lawson ML, et al. Closing the gap: results of the multicenter Canadian randomized controlled trial of structured transition in young adults with type 1 diabetes. Diabetes Care 2019;42:1018-26. 